\title{
Central Odontogenic Myxoma: A Radiographic Analysis
}

\author{
Ahmad Badruddin Ghazali $\mathbb{D},{ }^{1}$ Raweewan Arayasantiparb $\mathbb{D},{ }^{1}$ Rachai Juengsomjit $\mathbb{D}{ }^{2}$ \\ and Aroonwan Lam-ubol iD ${ }^{3}$ \\ ${ }^{1}$ Department of Oral and Maxillofacial Radiology, Faculty of Dentistry, Mahidol University, Bangkok 10400, Thailand \\ ${ }^{2}$ Department of Oral and Maxillofacial Pathology, Faculty of Dentistry, Mahidol University, Bangkok 10400, Thailand \\ ${ }^{3}$ Department of Oral Surgery and Oral Medicine, Faculty of Dentistry, Srinakharinwirot University, Bangkok 10110, Thailand
}

Correspondence should be addressed to Raweewan Arayasantiparb; raweewan.ara@mahidol.edu

Received 7 April 2021; Revised 2 June 2021; Accepted 14 June 2021; Published 28 June 2021

Academic Editor: Zohaib Khurshid

Copyright (c) 2021 Ahmad Badruddin Ghazali et al. This is an open access article distributed under the Creative Commons Attribution License, which permits unrestricted use, distribution, and reproduction in any medium, provided the original work is properly cited.

\begin{abstract}
Objective. This study aimed to determine the radiographic characteristics of odontogenic myxomas (OMs) and their associations. Materials and Methods. The study enrolled radiographs of patients taken between 2005 and 2019 with a confirmed histopathological diagnosis of central OM. OM radiographic features were evaluated, including location, border, locularity, involved area, the number of included teeth, root resorption, tooth displacement, bone expansion, bone perforation, and periosteal reaction. Fisher's exact test was used for statistical analysis. Results. Significant associations were found between the OM border and the affected jaw $(p=0.036)$, locularity $(p=0.036)$, involved areas $(p=0.009)$, and bone perforation $(p=0.036)$. OMs with an ill-defined border were associated with maxillary lesions, multilocularity, dentate areas, and cortical bone perforation. The number of included teeth ( 2 or fewer or 3 or more) was significantly associated with locularity $(p=0.010)$, involved area $(p=0.045)$, and bone expansion $(p=0.010)$. Larger OMs including 3 or more teeth, were associated with a multilocular appearance, dentate areas, and bone expansion. Conclusion. The border of $\mathrm{OM}$ and the number of included teeth are related to other radiographic appearances. Understanding these relationships could help in treatment decisions and help better understand the nature of OM.
\end{abstract}

\section{Introduction}

The World Health Organization classified odontogenic myxoma (OM) as a benign mesenchymal odontogenic tumor in 2017 [1]. It is rare, with a reported annual incidence of 0.07 per million [2], constituting approximately $1.9 \%-$ $6.3 \%$ of all odontogenic tumors [3-5]. The terms myxoma and myxofibroma can be used interchangeably, but when collagen fibers are prominent, the term myxofibroma is appropriate $[1,6]$. Although OMs grow slowly, they are invasive and infiltrate the local surrounding bone.

Most OMs are diagnosed in patients during the second to fourth decades of life [7-11]. They are common in the mandible, especially in the posterior region $[2,8,12,13]$. The OM border in panoramic images can be well-defined (with or without cortication) or ill-defined. OMs exhibit simple to aggressive radiographic manifestations: from a unilocular lesion at the periapical area or surrounding an unerupted tooth $[9,14]$ to a multilocular lesion mimicking ameloblastoma or odontogenic keratocyst [15], and including a sunburst appearance similar to osteosarcoma [16, 17]. They may displace teeth and cause root resorption. These various radiographic manifestations can lead to an initial misdiagnosis in patients with OM. The current study aimed to evaluate the radiographic characteristics of OM and the interrelationships among them.

\section{Materials and Methods}

The Institutional Review Board, Faculty of Dentistry/Faculty of Pharmacy, Mahidol University reviewed and approved the current cross-sectional study (MU-DT/PY-IRB 2019/ 025.0205). The inclusion criteria were patients with a confirmed histopathological diagnosis of central OM between January 2005 and December 2019 at the Oral and Maxillofacial Pathology Department, Faculty of Dentistry, 
Mahidol University. Patients for whom radiographs were unavailable were excluded. A board-certified-oral pathologist (R. J.) reviewed the hematoxylin and eosin-stained sections to confirm the histopathological diagnosis. In total, 12 patients were included in the study.

Panoramic radiographs were used to evaluate radiographic characteristics, including location, border, locularity, involved area, the number of included teeth, root resorption, and tooth displacement. Bone expansion and perforation were evaluated from occlusal cross-sectional, cone beam computed tomography (CBCT), or multidetector computed tomography (MDCT) images, depending on the availability of the imaging modalities for each patient. For evaluation of periosteal reaction, a panoramic radiograph combined with occlusal cross-sectional, CBCT, or MDCT images was used. Panoramic films of three patients were taken with a PM 2002 EC Proline (Planmeca, Helsinki, Finland). Digital panoramic radiographs were taken with a Kodak 9000 C (Carestream Health, Rochester, NY, USA). Ultraspeed or Insight (Eastman Kodak, Rochester, NY) occlusal cross-sectional films were exposed with a GX 1000 (Gendex, IL, USA) or Searcher Dx-068 (Belmont, Osaka, Japan). Occlusal cross-sectional digital radiographs were taken with a Planmeca ProX (Planmeca, Helsinki, Findland) using a phosphor plate system (VistaScan ${ }^{\circledR}$, Dürr Dental, Bietigheim-Bissingen, Germany). All radiographic films were digitized by scanning with a Microtek ScanMarker 9800XL (Microtek Inc, Santa Fe Spring, CA, USA) with a resolution of $300 \mathrm{dpi}$. The CBCT imaging was performed with a $3 \mathrm{D}$ Accuitomo (J. Morita, Kyoto, Japan) and a CB MercuRay (Hitachi Medical Systems, Tokyo, Japan). One MDCT imaging was performed with a Toshiba Alexion (Toshiba Medical Systems, Tochigi, Japan). All radiographs were evaluated using Picture Archiving and Communication System software installed on a computer running Microsoft Windows 10 (Microsoft Corp., Redmond, WA, USA) and displayed using RadiForce RX430 EIZO (EizoNanao Corporation, Ishikawa, Japan) on a 29.8-inch monitor $(2560 \times 1600$ pixels $)$. All radiographs were examined independently on a computer screen by a board-certified oral and maxillofacial radiologist (R.A.) and a radiologist in training (A. B. G.) in a dimly lit room. The correlation coefficient for the interobserver agreement was 0.99. Any disagreement between the two observers was resolved by consensus.

The location was classified as either anterior (i.e., region from central incisors to canines) or posterior (i.e., region from the first premolar to the tuberosity or ramus). The border was classified into three types: well-defined corticated, well-defined noncorticated, and ill-defined. The border was considered well-defined when an imaginary line could trace the limit of the lesion at the periphery. A corticated border was recorded when a thin, radiopaque line was present at the lesion's periphery. An ill-defined border represented the lesion with an indistinct marginal outline.

Locularity was categorized into unilocular and multilocular. A lesion was considered unilocular when it exhibited a single radiolucent area without any septa; it was considered multilocular when it exhibited at least two compartments with internal septa. Concerning the involved area, lesion presence in dentate, edentulous, or unerupted tooth areas was recorded. The number of included teeth was categorized into 2 types: OM, including two or fewer teeth and three or more teeth. Root resorption and displacement of adjacent teeth were also documented. Bucco-lingual bone expansion, cortical bone perforation, and periosteal reaction were also recorded.

Descriptive statistics were used to describe radiographic features. The Fisher's exact test was used to analyze the association between radiographic features using IBM SPSS Statistics for Windows (Version 21.0., IBM Corp., Armonk, NY, USA). A $p<0.05$ was considered statistically significant.

\section{Results}

Among the 658 patients with odontogenic tumors who presented at the Department of Oral and Maxillofacial Pathology over the 15 year study period, 15 (2.3\%) had OMs, and 13 (2\%) had central OMs. The study included 12 patients who had both panoramic images and occlusal cross-sectional, CBCT or MDCT images.

Of the 12 patients, four were men, and eight were women. Patient age ranged between 21 and 51 years (mean age, $32.4 \pm 10$ years). OMs were found incidentally in the panoramic radiographs of five asymptomatic patients. Six patients presented with the chief complaint of painless swelling in the affected area; the remaining patients complained of gingival abscess in the involved area. Eight patients $(66.7 \%)$ exhibited a mandibular lesion, while four (33.3\%) exhibited a maxillary lesion. Lesions were in the posterior region in eight patients, in the anterior-posterior region in three, and in the anterior region in one. Midlinecrossing lesions were found in two patients: one in the maxilla and the other in the mandible.

The lesion border was well-defined corticated in three patients (25\%; all mandibular lesions), well-defined noncorticated in six (50\%; five mandibular lesions and one maxillary lesion), and ill-defined in three (25\%; all maxillary lesions) (Figure 1). Most OMs (8/12) showed multilocular radiolucency, while four showed unilocular radiolucency. Three unilocular lesions were associated with an unerupted lower third molar (Figure 2). OMs mainly involved the dentate area in nine patients (75\%) and unerupted teeth in three (25\% - all unilocular OMs). The superior portion of the lesion in all 12 patients was located near the alveolar crest bone. Five OMs (41.7\%) included the area of 2 or fewer teeth, and seven OMs (58.3\%) included the area of 3 or more teeth. Displacement of adjacent teeth was present in six patients (50\%), and root resorption of involved teeth was present in three patients $(25 \%)$. The bone expansion was found in 8 OMs (66.7\%); however, bone perforation was found in 4 OMs (33.3\%). All perforation cases had a multilocular appearance, occurred in dentate areas, and had a lesional size, including the area of 3 or more teeth (Figure 3(a)). By comparison, all OMs with unerupted teeth were unilocular with no finding of bone perforation and having a lesional size of OMs including 2 or fewer teeth (Figure 3(b)). There was no periosteal reaction in any of the OMs. The recorded 


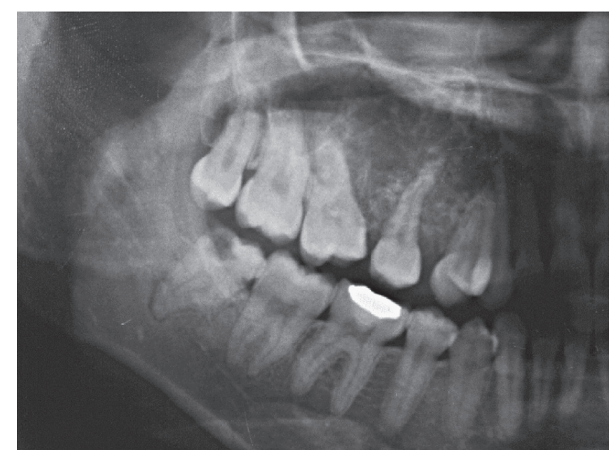

FIGURE 1: Maxillary odontogenic myxoma in a 26-year-old woman (Patient 3). Cropped panoramic image shows ill-defined multilocular radiolucency located in the dentate area from maxillary right first premolar to the third molar.

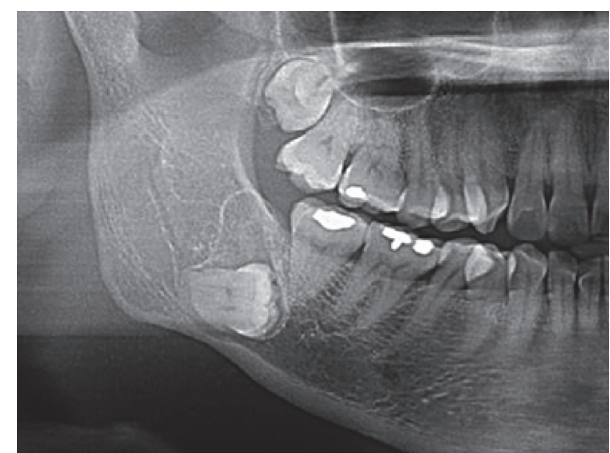

FIGURE 2: Mandibular odontogenic myxoma in a 44-year-old woman (Patient 9). Cropped panoramic image shows well-defined corticated, unilocular radiolucency associated with unerupted mandibular right third molar.

radiographic characteristics are summarized in Tables 1 and 2.

Table 3 shows the relationships between the OM borders and jaws $(p=0.036)$, locularity $(p=0.036)$, the involved area $(p=0.009)$, and bone perforation $(p=0.036)$. All OMs with ill-defined borders were maxillary cases, multilocular in appearance, presenting in dentate areas, and having bone perforation. The study also revealed associations between the number of included teeth and locularity $(p=0.010)$, involved area $(p=0.045)$, and bone expansion $(p=0.010)$ in Table 4 . All OMs, including area 3 or more teeth, presented multilocularity, occurred in dentate areas, and had bone expansion. There was, however, no association between the OM border and the number of included teeth of OMs. Besides the foregoing, no other association with radiographic features was found.

\section{Discussion}

During the study period, a respective $2.3 \%$ and $2 \%$ of all patients diagnosed with odontogenic tumors at our institute had OMs and central OMs. Our findings confirm that OMs are rare and are consistent with an OM incidence of approximately $1.9 \%$ of all odontogenic tumors in Asians $[4,5]$. $\mathrm{OM}$ is, however, the second most common odontogenic tumor with a frequency of between $10.3 \%$ and $19 \%$ of all odontogenic tumors in Africa $[18,19]$, which might be due to a regional or ethnic difference.

In the current study, OMs occurred more frequently in women (male-to-female ratio of $1: 2$ ) as in several studies (male-to-female ratio ranging between $1: 1.8$ and $1: 4.8$ ) $[2,5,7-10,12,20-22]$. A male predilection was, however, observed in some studies $[13,23,24]$. Although the age at diagnosis of OM varies, most OMs are observed in patients during the second to fourth decade of life [2, 8-11], including in our study. The peak age of diagnosis was during the third decade of life ( $50 \%$ of patients in the current study), as was found in several previous studies [8-10, 22, 24]. Manila et al. reported that the mean age for male patients (56.7 years) was greater than that of female patients (40.5 years) [23]; by comparison, we found the respective age was 34 and 31.6 years.

Clinical complaints vary among studies. Some studies reported that swelling was the most common clinical complaint of patients with OM [7, 8], while Simon et al. reported that no clinical symptoms were observed in most patients with OM [2]. In the present study, half of the patients presented with the chief complaint of painless swelling in the affected area. Furthermore, $41.7 \%$ of lesions were found incidentally on panoramic radiographs taken during routine dental examinations. No patients had pain in our study, whereas pain was present in $25 \%-28 \%$ of patients in some studies $[2,8]$.

Our findings support the notion that $\mathrm{OM}$ is more common in the mandible $[2,7,8,22]$, as was the case for two-thirds of our patients. Some studies, however, found equal incidences of OMs in both the maxilla and mandible $[5,13,25]$. The predominant area of the jaw affected by OM is reportedly the posterior region, especially the premolarmolar region $[2,13]$ or the posterior mandible and ramus $[22,26]$. In our study, two-thirds of our cases exhibited lesions in the posterior region, while three patients (25\%) exhibited lesions in the anterior-posterior region. Only one patient exhibited a lesion in the anterior region alone, and this lesion also crossed the midline in the maxilla. These findings agree with the results reported by Takahashi et al., who found that all OMs in the anterior region were maxillary OMs [5]. Of note, we found two midline-crossing lesions $(16.7 \%)$, both of which were multilocular OMs, one in the maxilla and the other in the mandible. The midline-crossing mandibular lesion was a large OM for which involvement extended from the left premolar region to the right molar region. Similarly, most OMs did not cross the midline in prior reports $[5,27]$. A summary of the demographic and radiographic findings of patients in large case series reported in the English-language medical literature in recent decades is shown in Table 5.

In the current study, $25 \%$ and $50 \%$ of patients had welldefined corticated and noncorticated OM borders, respectively. One-quarter of patients had ill-defined OM borders. Our findings are consistent with previous studies in which well-defined borders were present in between $58 \%$ and $84 \%$ of patients, while ill-defined borders were present in between $16 \%$ and $42 \%$ of patients $[9,20,27]$. We found a significant 


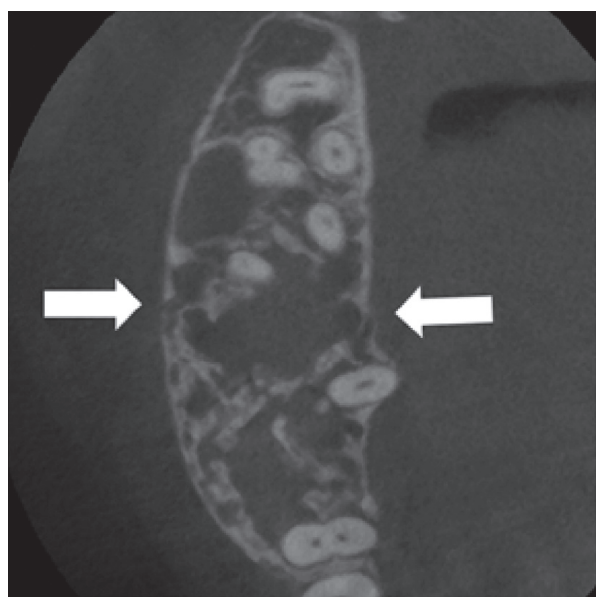

(a)

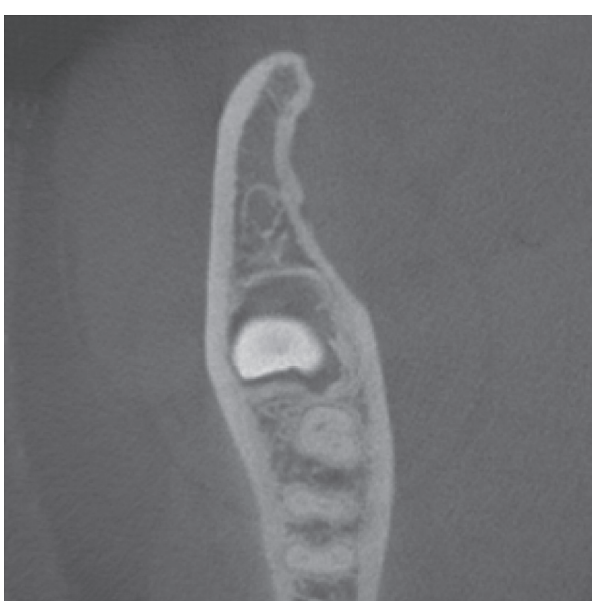

(b)

Figure 3: Axial CBCT images. (a) Maxillary odontogenic myxoma (Patient 3) at the dentate area, including more than 3 teeth with bone expansion and perforation (arrows). (b) Mandibular odontogenic myxoma (Patient 9) with unerupted third molar without bone perforation.

TABLE 1: Radiographic features of 12 patients with central odontogenic myxoma-location, border, locularity, involved area, and the number of included teeth.

\begin{tabular}{lcccccccc}
\hline Patient & Sex & Age & Jaw & Location & Border & Locularity & Involved area & Number of included teeth \\
\hline 1 & $F$ & 34 & Maxilla & $R$ post & Ill & Multilocular & Dentate area & $\geq 3$ \\
2 & $M$ & 31 & Maxilla & $L$ ant to $L$ post & Ill & Multilocular & Dentate area & $\geq 3$ \\
3 & $F$ & 26 & Maxilla & $R$ post & Ill & Multilocular & Dentate area & $\geq 3$ \\
4 & $M$ & 32 & Maxilla & $R$ ant to $L$ ant & WDNC & Multilocular & Dentate area & $\geq 3$ \\
5 & $F$ & 21 & Mandible & $R$ post & WDNC & Unilocular & Dentate area & $\leq 2$ \\
6 & $F$ & 23 & Mandible & $R$ post & WDNC & Multilocular & Dentate area & $\leq 3$ \\
7 & $F$ & 27 & Mandible & $L$ post & WDC & Unilocular & Third molar unerupted & $\leq 2$ \\
8 & $M$ & 48 & Mandible & $R$ post $L$ post & WDNC & Multilocular & Dentate area & $\leq 3$ \\
9 & $F$ & 44 & Mandible & $R$ post & WDC & Unilocular & Third molar unerupted & $\leq 2$ \\
10 & $F$ & 51 & Mandible & $L$ post & WDC & Unilocular & Third molar unerupted & $\leq 2$ \\
11 & $F$ & 27 & Mandible & $R$ ant to $R$ post & WDNC & Multilocular & Dentate area & $\leq 2$ \\
12 & $M$ & 25 & Mandible & $R$ post & WDNC & Multilocular & Dentate area & \\
\hline
\end{tabular}

$R$ : right; $L$ : left; Post: posterior; Ant: anterior; WDC: well-defined corticated; WDNC: well-defined noncorticated; Ill: ill-defined.

TABLE 2: Radiographic features of 12 patients with central odontogenic myxoma-root resorption, tooth displacement, bone expansion, bone perforation, and periosteal reaction.

\begin{tabular}{|c|c|c|c|c|c|c|c|}
\hline Patient & Sex & Age & Root resorption & Tooth displacement & Bone expansion & Bone perforation & Periosteal reaction \\
\hline 1 & $F$ & 34 & No & Yes & Yes & Yes & No \\
\hline 2 & $M$ & 31 & No & Yes & Yes & Yes & No \\
\hline 3 & $F$ & 26 & No & Yes & Yes & Yes & No \\
\hline 4 & $M$ & 32 & No & Yes & Yes & No & No \\
\hline 5 & $F$ & 21 & Yes & Yes & No & No & No \\
\hline 6 & $F$ & 23 & No & No & Yes & No & No \\
\hline 7 & $F$ & 27 & No & No & Yes & No & No \\
\hline 8 & $M$ & 48 & Yes & Yes & Yes & Yes & No \\
\hline 9 & $F$ & 44 & No & No & No & No & No \\
\hline 10 & $F$ & 51 & No & No & No & No & No \\
\hline 11 & $F$ & 27 & Yes & No & Yes & No & No \\
\hline 12 & $M$ & 25 & No & No & No & No & No \\
\hline
\end{tabular}


TABLE 3: Relationships of odontogenic myxoma borders with jaws, locularity, involved area, bone perforation, bone expansion, number of included teeth, root resorption, and tooth displacement.

\begin{tabular}{|c|c|c|c|c|}
\hline & \multicolumn{3}{|c|}{ Border $(n=12)$} & \multirow{2}{*}{$p$ value } \\
\hline & WDC & WDNC & Ill & \\
\hline \multicolumn{5}{|l|}{ Jaw } \\
\hline Maxilla & 0 & 1 & 3 & $0.036^{*}$ \\
\hline Mandible & 3 & 5 & 0 & \\
\hline \multicolumn{5}{|l|}{ Locularity } \\
\hline Unilocular & 3 & 1 & 0 & $0.036^{*}$ \\
\hline Multilocular & 0 & 5 & 3 & \\
\hline \multicolumn{5}{|l|}{ Involved area } \\
\hline Unerupted tooth & 3 & 0 & 0 & $0.009^{*}$ \\
\hline Dentate area & 0 & 6 & 3 & \\
\hline \multicolumn{5}{|l|}{ Bone perforation } \\
\hline Yes & 0 & 1 & 3 & $0.036^{*}$ \\
\hline No & 3 & 5 & 0 & \\
\hline \multicolumn{5}{|l|}{ Bone expansion } \\
\hline Yes & 1 & 4 & 3 & 0.267 \\
\hline No & 2 & 2 & 0 & \\
\hline \multicolumn{5}{|c|}{ Number of included teeth } \\
\hline 2 or fewer teeth & 3 & 2 & 0 & 0.053 \\
\hline 3 or more teeth & 0 & 4 & 3 & \\
\hline \multicolumn{5}{|l|}{ Root resorption } \\
\hline Yes & 0 & 3 & 0 & 0.345 \\
\hline No & 3 & 3 & 3 & \\
\hline \multicolumn{5}{|l|}{ Tooth displacement } \\
\hline Yes & 0 & 3 & 3 & 0.123 \\
\hline No & 3 & 3 & 0 & \\
\hline
\end{tabular}

WDC: well-defined corticated; WDNC: well-defined noncorticated; Ill: illdefined. *Significant relationship $(p<0.05)$.

association between the OM border and the affected jaw. Most maxillary lesions had ill-defined borders, while all mandibular lesions had well-defined borders. These findings agree with other studies wherein maxillary OMs are typically ill-defined, while mandibular OMs are typically well-defined $[9,28]$.

The present study revealed an association between the $\mathrm{OM}$ border and locularity. All OMs with an ill-defined border presented multilocularity. All unilocular lesions had well-defined borders. Although OMs can have a unilocular or multilocular presentation, most (66.7\%) OMs in the current study were multilocular, as reported by several other studies [2, 8-10, 12, 22, 24, 29]. Takahashi et al. found comparable proportions of unilocular and multilocular lesions-all unilocular lesions were in the maxilla, while all multilocular lesions were in the mandible [5]. While Keszler et al. found that OMs were predominantly unilocular [13], one-third of OMs in our study exhibited unilocular radiolucency-all of which were mandibular OMs.

OMs in our study mainly involved dentate areas in nine patients $(75 \%)$ and unerupted teeth in three $(25 \%$; all unilocular) as with previous reports of a few OM cases found with unerupted teeth [27]. An association between the OM border and the involved area was also observed in our study. All OMs with unerupted teeth had well-defined, corticated borders. An association was also found between the OM border and bone perforation. Most cases with bone perforation had an ill-defined border. All cases without bone
TABLE 4: Relationships between the number of included teeth of odontogenic myxoma and jaw, locularity, involved area, bone perforation, bone expansion, border, root resorption, and tooth displacement.

\begin{tabular}{lccc}
\hline & $\begin{array}{c}\text { Included teeth } \\
(n=12)\end{array}$ & $p$ value \\
& $\leq 2$ teeth $\geq 3$ teeth & \\
\hline Jaw & 0 & 4 & 0.081 \\
$\quad$ Maxilla & 5 & 3 & \\
$\quad$ Mandible & & & \\
Locularity & 4 & 0 & $0.010^{*}$ \\
$\quad$ Unilocular & 1 & 7 & \\
$\quad$ Multilocular & & & \\
Involved area & 3 & 0 & $0.045^{*}$ \\
$\quad$ Unerupted tooth & 2 & 7 & \\
$\quad$ Dentate area & & & \\
Bone perforation & 0 & 4 & 0.081 \\
$\quad$ Yes & 5 & 3 & \\
$\quad$ No & & & \\
Bone expansion & 1 & 7 & $0.010^{*}$ \\
$\quad$ Yes & 4 & 0 & \\
$\quad$ No & & & \\
Border & 3 & 0 & 0.053 \\
$\quad$ Well-defined corticated & 2 & 4 & \\
$\quad$ Well-defined noncorticated & 0 & 3 & \\
$\quad$ Ill & & & \\
Root resorption & 1 & 2 & 1.000 \\
$\quad$ Yes & 4 & 5 & \\
$\quad$ No & & & \\
Tooth displacement & & 5 & 0.242 \\
$\quad$ Yes & & & \\
$\quad$ No & & & \\
\hline
\end{tabular}

${ }^{*}$ Significant relationship $(p<0.05)$.

perforation had a well-defined border. Although we did not find any previous reports of an association between cortical perforation and OM border, cortical perforation was reportedly associated with large-sized OMs [15]. We were not able to confirm this finding.

OMs including 2 or fewer teeth or 3 or more teeth were significantly associated with the locularity in our study. All unilocular cases were OMs including 2 or fewer teeth, while almost multilocular cases were OMs including 3 or more teeth. Our finding confirms a previous result indicating that OMs with a unilocular appearance tend to include a smaller area than OMs with a multilocular appearance $[14,15,20,27]$. This observation was supported by Kauke et al. [15], who found that large-sized OMs were associated with multilocularity, so they suggested that multilocularity was one of the signs of aggressiveness in OM. A systematic study in 2020 also found that most of the recurrences reported were multilocular in appearance [12].

OMs including 2 or fewer teeth or 3 or more teeth were also associated with the involved area. All OMs with unerupted teeth were small OMs including 2 or fewer teeth, while most OMs at the dentate area were larger OMs including 3 or more teeth. One previous study reported a large $\mathrm{OM}$ with an unerupted tooth [21]. No association has been reported between size and bone expansion or presentation of an unerupted tooth [30]. An association between OMs including 2 or 


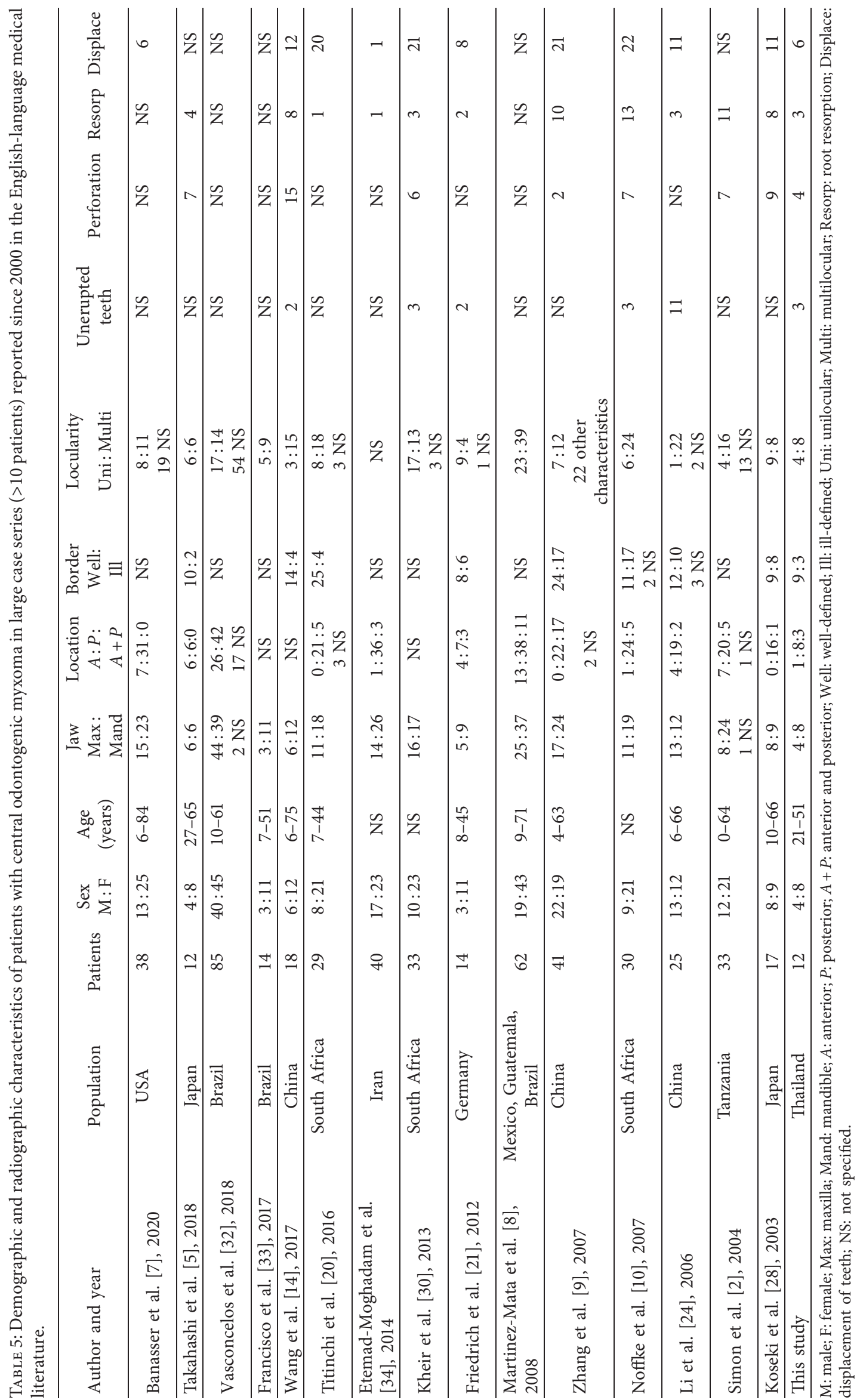


fewer teeth or 3 or more teeth and bone expansion was found in our study. Most expansion cases were large OMs including 3 or more teeth, while all nonexpansion cases were smaller OMs including 2 or fewer teeth.

A notable observation in our study was the superior aspect of all lesions-located near the alveolar crest. Regarding tooth displacement, previous studies found that approximately $20 \%$ of patients with OM exhibited tooth displacement $[2,8,22]$. By contrast, the present study found displacement of adjacent teeth in $50 \%$ of patients. Root resorption of involved teeth occurred in $25 \%$ of patients in the current study, all of whom had mandibular lesions. Previous studies similarly reported that root resorption was present in approximately $20 \%-50 \%$ of patients with OM $[2,5,22]$. As for periosteal reaction, we did not find any cases that showed this radiographic characteristic, although it has been reported as a sunray appearance in some reports $[9,14]$.

As for imaging evaluation of OM, panoramic radiography and computed tomography are sufficient [12], although Manila et al. suggest that magnetic resonance imaging should also be used to more accurately determine the margins [23]. Panoramic radiography is the most commonly used modality to evaluate OM [12]. Computed tomography gives more detailed information, which is useful for surgical treatment planning that can range from $\mathrm{cu}^{-}$ rettage to extensive resection, requiring a multidisciplinary approach for rehabilitation [31].

A limitation of the study was the small number of patients due to the rarity of the tumor. Another limitation was that the $3 \mathrm{D}$ volume images were not available for all of the cases. Additional investigations with a larger number of patients and advanced imaging are needed to better understand the nature of the lesion. Further studies to identify the association between $3 \mathrm{D}$ images and histopathogenesis-especially using immunohistochemistry-are recommended to better understand the pathogenesis and aggressiveness of some types of lesions.

\section{Conclusion}

OM has a variety of radiographic characteristics. OMs with an ill-defined border were associated with the maxilla, multilocularity, dentate areas, and bone perforation. Large OMs including 3 or more teeth were associated with multilocularity, dentate areas, and bone expansion. There was, however, no association between the OM border and the number of included teeth. Knowing the border and the included teeth (whether few or many) helps to understand the nature of the OM.

\section{Data Availability}

The research data used to support this study are included within the article.

\section{Conflicts of Interest}

The authors declare no conflicts of interest.

\section{Acknowledgments}

The authors thank the staff at the Department of Oral and Maxillofacial Radiology and the Department of Oral and Maxillofacial Pathology, for assistance in data collection, and Assistant Professor Chulalak Komoltri, for assistance with the statistical analysis.

\section{References}

[1] E. W. Odell and K. Adebiyi, "Benign mesenchymal odontogenic tumours," in WHO Classification of Head and Neck Tumours, A. K. EI-Maggar, J. K. C. Chan, J. R. Grandis, T. Takata, and P. J. Slootweg, Eds., pp. 229-230, IARC, Lyon, France, 4th edition, 2017.

[2] E. N. M. Simon, M. A. W. Merkx, E. Vuhahula, D. Ngassapa, and P. J. W. Stoelinga, "Odontogenic myxoma: a clinicopathological study of 33 cases," International Journal of Oral and Maxillofacial Surgery, vol. 33, no. 4, pp. 333-337, 2004.

[3] B. S. M. S. Siriwardena, H. Crane, N. O’Neill et al., "Odontogenic tumors and lesions treated in a single specialist oral and maxillofacial pathology unit in the United Kingdom in 1992-2016," Oral Surgery, Oral Medicine, Oral Pathology and Oral Radiology, vol. 127, no. 2, pp. 151-166, 2019.

[4] H. Hosgor, B. Tokuc, B. Kan, and F. M. Coskunses, "Evaluation of biopsies of oral and maxillofacial lesions: a retrospective study," Journal of the Korean Association of Oral and Maxillofacial Surgeons, vol. 45, no. 6, pp. 316-323, 2019.

[5] Y. Takahashi, K. Tanaka, H. Hirai, E. Marukawa, T. Izumo, and H. Harada, "Appropriate surgical margin for odontogenic myxoma: a review of 12 cases," Oral Surgery, Oral Medicine, Oral Pathology and Oral Radiology, vol. 126, no. 5, pp. 404408, 2018.

[6] B. F. Barker, "Odontogenic myxoma," Seminars in Diagnostic Pathology, vol. 16, no. 4, pp. 297-301, 1999.

[7] A. M. Banasser, M. M. Bawazir, M. N. Islam, I. Bhattacharyya, D. M. Cohen, and S. G. Fitzpatrick, "Odontogenic myxoma: a 23-year retrospective series of 38 cases," Head and Neck Pathology, vol. 14, no. 4, pp. 1021-1027, 2020.

[8] G. Martínez-Mata, A. Mosqueda-Taylor, R. Carlos-Bregni et al., "Odontogenic myxoma: clinico-pathological, immunohistochemical and ultrastructural findings of a multicentric series," Oral Oncology, vol. 44, no. 6, pp. 601-607, 2008.

[9] J. Zhang, H. Wang, X. He, Y. Niu, and X. Li, "Radiographic examination of 41 cases of odontogenic myxomas on the basis of conventional radiographs," Dentomaxillofacial Radiology, vol. 36, no. 3, pp. 160-167, 2007.

[10] C. E. E. Noffke, E. J. Raubenheimer, N. J. Chabikuli, and M. M. R. Bouckaert, "Odontogenic myxoma: review of the literature and report of 30 cases from South Africa," Oral Surgery, Oral Medicine, Oral Pathology, Oral Radiology, and Endodontics, vol. 104, no. 1, pp. 101-109, 2007.

[11] S. O. Ajike, U. E. Amanyeiwe, and E. O. Adekeye, "Myxoma of the jaw bones: analysis of 27 cases," Nigerian Journal of Surgical Research, vol. 2, no. 3, pp. 123-126, 2000.

[12] J. H. Dotta, L. N. Miotto, R. Spin-Neto, and T. M. Ferrisse, "Odontogenic myxoma: systematic review and bias analysis," European Journal of Clinical Investigation, vol. 50, no. 4, Article ID e13214, 2020.

[13] A. Keszler, F. V. Dominguez, and G. Giannunzio, "Myxoma in childhood," Journal of Oral and Maxillofacial Surgery, vol. 53, no. 5, pp. 518-521, 1995.

[14] K. Wang, W. Guo, M. You, L. Liu, B. Tang, and G. Zheng, "Characteristic features of the odontogenic myxoma on cone 
beam computed tomography," Dentomaxillofacial Radiology, vol. 46, no. 2, p. 20160232, 2017.

[15] M. Kauke, A.-F. Safi, M. Kreppel et al., "Size distribution and clinicoradiological signs of aggressiveness in odontogenic myxoma-three-dimensional analysis and systematic review," Dentomaxillofacial Radiology, vol. 47, no. 2, p. 20170262, 2018.

[16] J. A. White, N. Ramer, T. R. Wentland, and M. Cohen, "The rare radiographic sunburst appearance of odontogenic myxomas: a case report and review of the literature," Head and Neck Pathology, vol. 14, no. 4, pp. 1105-1110, 2020.

[17] S. Goel, M. Goel, and A. D. Dinkar, "Odontogenic myxoma of mandible with unusual (sunburst) appearance: a rare case report," Journal of Clinical and Diagnostic Research, vol. 10, no. 5, pp. ZJ05-ZJ07, 2016.

[18] E. N. Simon, P. J. Stoelinga, E. Vuhahula, and D. Ngassapa, "Odontogenic tumours and tumour-like lesions in Tanzania," East African Medical Journal, vol. 79, no. 1, pp. 3-7, 2002.

[19] E. T Adebayo, S. O. Ajike, and E. O. Adekeye, "Odontogenic tumours in children and adolescents: a study of 78 Nigerian cases," Journal of Cranio-Maxillofacial Surgery, vol. 30, no. 5, pp. 267-272, 2002.

[20] F. Titinchi, B. A. Hassan, J. A. Morkel, and C. Nortje, "Odontogenic myxoma: a clinicopathological study in a South African population," Journal of Oral Pathology and Medicine, vol. 45, no. 8, pp. 599-604, 2016.

[21] R. E. Friedrich, H. A. Scheuer, A. Fuhrmann, J. Zustin, and A. T. Assaf, "Radiographic findings of odontogenic myxomas on conventional radiographs," Anticancer Research, vol. 32, no. 5, pp. 2173-2177, 2012.

[22] L. L. Muzio, P. Nocini, G. Favia, M. Procaccini, and M. D. Mignogna, "Odontogenic myxoma of the jaws A clinical, radiologic, immunohistochemical, and ultrastructural study," Oral Surgery, Oral Medicine, Oral Pathology, Oral Radiology, and Endodontics, vol. 82, no. 4, pp. 426-433, 1996.

[23] N. G. Manila, J. Sakamoto, T. Kurabayashi et al., "Odontogenic myxoma: a case report and literature review," Oral Surgery, Oral Medicine, Oral Pathology and Oral Radiology, vol. 130, no. 2, pp. e64-e65, 2020.

[24] T.-J. Li, L.-S. Sun, and H.-Y. Luo, "Odontogenic myxoma: a clinicopathologic study of 25 cases," Archives of Pathology and Laboratory Medicine, vol. 130, no. 12, pp. 1799-1806, 2006.

[25] S. S. Subramaniam, A. A. Heggie, R. Kumar, and J. M. Shand, "Odontogenic myxoma in the paediatric patient: a review of eight cases," International Journal of Oral and Maxillofacial Surgery, vol. 45, no. 12, pp. 1614-1617, 2016.

[26] P. Boffano, C. Gallesio, A. Barreca, F. A. Bianchi, P. GarzinoDemo, and F. Roccia, "Surgical treatment of odontogenic myxoma," Journal of Craniofacial Surgery, vol. 22, no. 3, pp. 982-987, 2011.

[27] I. Kaffe, H. Naor, and A. Buchner, "Clinical and radiological features of odontogenic myxoma of the jaws," Dentomaxillofacial Radiology, vol. 26, no. 5, pp. 299-303, 1997.

[28] T. Koseki, K. Kobayashi, K. Hashimoto et al., "Computed tomography of odontogenic myxoma," Dentomaxillofacial Radiology, vol. 32, no. 3, pp. 160-165, 2003.

[29] R. Worawongvasu and M. Tiensuwan, "Odontogenic tumors in Thailand: a study of 590 Thai patients," Journal of Oral and Maxillofacial Surgery, Medicine, and Pathology, vol. 27, no. 4, pp. 567-576, 2015.

[30] E. Kheir, L. Stephen, C. Nortje, L. Janse van Rensburg, and F. Titinchi, "The imaging characteristics of odontogenic myxoma and a comparison of three different imaging modalities," Oral Surgery, Oral Medicine, Oral Pathology and Oral Radiology, vol. 116, no. 4, pp. 492-502, 2013.

[31] I. Smojver, M. Vuletić, S. Manojlović, and D. Gabrić, "Multidisciplinary approach to rehabilitation after tumor resective jaw surgery: a 9 year follow-up," Case Reports in Dentistry, vol. 2020, Article ID 8867320, , 2020.

[32] A. C. U. Vasconcelos, F. M. Silveira, A. P. N. Gomes et al., "Odontogenic myxoma: a 63-year retrospective multicenter study of 85 cases in a Brazil population and a review of 999 cases from literature," Journal of Oral Pathology and Medicine, vol. 47, no. 1, pp. 71-77, 2018.

[33] A. L. Francisco, T. C. Chulam, F. O Silva et al., "Clinicopathologic analysis of 14 cases of odontogenic myxoma and review of the literature," Journal of Clinical and Experimental Dentistry, vol. 9, no. 4, pp. e560-e563, 2017.

[34] S. Etemad-Moghadam, S. Chookhachizadeh, F. Baghaii, and M. Alaeddini, "Odontogenic Myxoma: a study based on biopsy material over a 40-year period," The Journal of Contemporary Dental Practice, vol. 15, no. 2, pp. 137-141, 2014. 